\title{
CONVERGENT CLOSE-COUPLING APPROACH TO ELECTRON-ATOM COLLISIONS
}

\author{
Igor Bray and Andris Stelbovics \\ ARC Centre for Antimatter-Matter Studies, Murdoch University, Perth 6150, Australia
}

\begin{abstract}
The Temkin-Poet model of electron-hydrogen scattering has played a crucial role in the development of many general computational methods for collisions in atomic physics, and in particular the convergent close-coupling (CCC) method. Here we review the CCC method to electron-atom scattering and give the historical perspective of its development utilising the Temkin-Poet model.
\end{abstract}

\section{INTRODUCTION}

It was with great pleasure and honour to accept the invitation to make a presentation at the symposium celebrating the life-long work of Aaron Temkin and Richard Drachman. The work of Aaron Temkin was particularly influential on our own during the development of the CCC method for electron-atom collisions. There are a number of key problems that need to be dealt with when developing a general computational approach to such collisions. Traditionally, the electron energy range was subdivided into the low, intermediate, and high energies. At the low energies only a finite number of channels are open and variational or close-coupling techniques could be used to obtain accurate results [1,2]. At high energies an infinite number of discrete channels and the target continuum are open, but perturbative techniques are able to yield accurate results [3]. However, at the intermediate energies perturbative techniques fail and computational approaches need to be found for treating the infinite number of open channels. In addition, there are also problems associated with the identical nature of electrons and the difficulty of implementing the boundary conditions for ionization processes.

The beauty of the Temkin-Poet model of electron-hydrogen scattering is that it simplifies the full computational problem by neglecting any non-zero orbital angular momenta in the partial-wave expansion, without loosing the complexity associated with the above-mentioned problems [4-9]. The unique nature of the problem allowed for accurate solution leading to benchmark results which could then be used to test the much more general approaches to electron-atom collision problems.

The immense value of the Temkin-Poet model is readily summarised by the fact that the initial papers of Temkin [4] and Poet [7] have been collectively cited around 250 times to date and are still being cited in present times [10, 11]. Many of the citations came from our own work during the course of the development of the CCC method, which we now describe.

\section{THE CONVERGENT CLOSE-COUPLING METHOD}

At the beginning of the 1990s there were major discrepancies between theory and experiment for the most fundamental electron atom collision systems such as e-H and e-He scattering. For $54.4 \mathrm{eV}$ electron-hydrogen excitation there were two independent measurements of the $2 p$ angular correlation parameters in broad agreement with each other $[12,13]$, but not with the most sophisticated calculations available at the time [14-17]. For electron-helium scattering, a favourite system for the experimentalists, the discrepancies were even much more widespread over the energy range and variety of excitation parameters. For both targets there was no ab initio description of the total ionization cross sections, measured with an uncertainty of around 5\% [18-20]. The CCC method was developed with the view to addressing these problems, starting with the e-H system.

The CCC method is based on the close-coupling method which expands the total e-H wavefunction $\Psi_{i}^{(+)}\left(\boldsymbol{r}_{1}, \boldsymbol{r}_{2}\right)$ using a set of known target-space states $\phi_{n}\left(\boldsymbol{r}_{2}\right)$. The effect of the Temkin-Poet model is to reduce the dimensionality of the problem by reducing the vector nature of the $\boldsymbol{r}$ coordinates to scalars $r$. In other words, angular dependence of the scattering is neglected. In the original Laguerre-based CCC method [21], the target states $\phi_{f}^{(\lambda)}(r)=\sum_{n=1}^{N} C_{f n} \xi_{n}^{(\lambda)}(r)$, where $\xi_{n}^{(\lambda)}(r)$ is a Laguerre basis with exponential fall-off factor $\lambda$, are obtained by diagonalising the hydrogen Hamiltonian

$$
\left\langle\phi_{f}^{(\lambda)}\left|H_{\mathrm{T}}\right| \phi_{i}^{(\lambda)}\right\rangle=\varepsilon_{f}^{(\lambda)} \delta_{f i}, \quad i, f=1, \ldots, N
$$

More recently [22], another way of obtaining the target states is by solving the eigenstate problem in a box

$$
H_{\mathrm{T}}\left|\phi_{i}^{\left(R_{0}\right)}\right\rangle=\varepsilon_{i}^{\left(R_{0}\right)}\left|\phi_{i}^{\left(R_{0}\right)}\right\rangle, \quad i=1, \ldots, N
$$




\section{Symposium on Atomic \& Molecular Physics}

for $r \leq R_{0}$, with $\phi_{i}^{\left(R_{0}\right)}(0)=\phi_{i}^{\left(R_{0}\right)}\left(R_{0}\right)=0$. We distinguish between the CCC method using the above two sets of states as CCC-L and CCC-B, respectively.

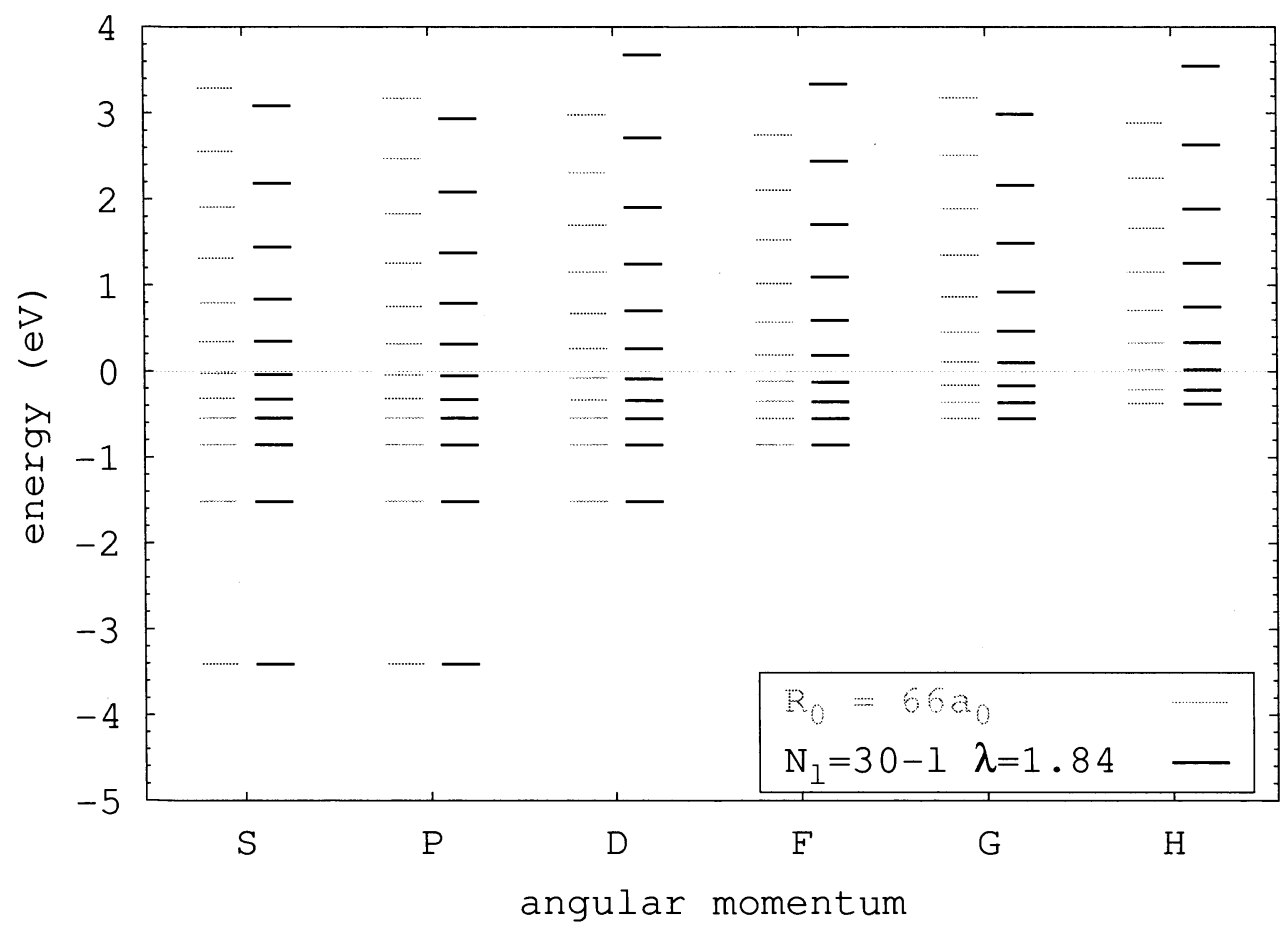

FIG. 1: Hydrogen excited-state energy levels in CCC-B $\left(R_{0}=66 a_{0}\right)$ and CCC-L $\left(N_{l}=30-l, \lambda=1.84\right)$ calculations.

In figure 1 we present the energy levels that can result from Laguerre-based (CCC-L) or box-based (CCC-B) calculations. The parameters specified were chosen in such a way so as to indicate that there is considerable similarity in the two approaches. The negative and low positive-energies are almost identical for all $l$, with variation occurring only for the higher energies.

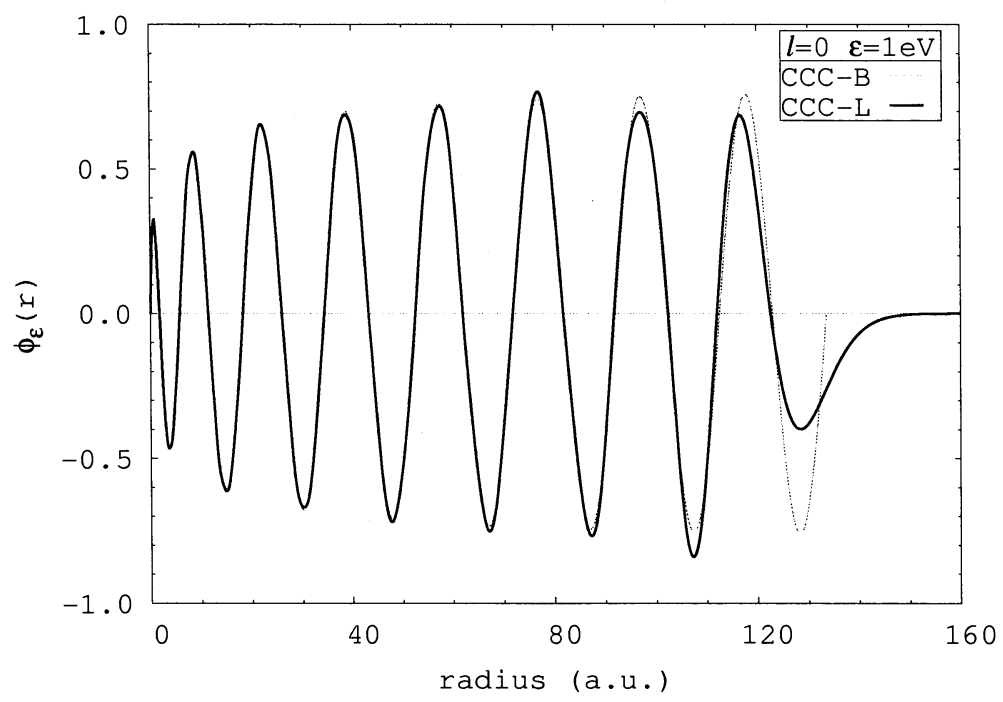

FIG. 2: CCC-B $(N=70, \lambda=2)$ and CCC-L $\left(R_{0}=134 a_{0}\right) 1 \mathrm{eV}$ suitably normalised wavefunctions for $l=0$.

Having looked at the energies, in figure 2 we compare the $1 \mathrm{eV}$ wavefunctions for $l=0$ arising from another set of 


\section{Symposium on Atomic \& Molecular Physics}

calculations. We see that the two functions are almost identical until the larger radial values, with the major variation being where the Laguerre-basis exponential fall-off dominates past $120 a_{0}$.

Once the states $\phi(r)$ are defined, utilising either approach, they are then used to expand the appropriately symmetrised total wavefunction

$$
\left|\Psi_{i}^{(+)}\right\rangle=\mathcal{A}\left|\psi_{i}^{(+)}\right\rangle \approx \mathcal{A} \sum_{n=1}^{N}\left|\phi_{n}\right\rangle\left\langle\phi_{n} \mid \psi_{i}^{(+)}\right\rangle .
$$

In the CCC method we write the resulting close-coupling equations in the form of coupled integral equations for the transition matrix $\left\langle k_{f} \phi_{f}|T| \phi_{i} k_{i}\right\rangle \equiv\left\langle k_{f} \phi_{f}|V| \Psi_{i}^{(+)}\right\rangle$, which satisfy

$$
\begin{aligned}
\left\langle k_{f} \phi_{f}|T| \phi_{i} k_{i}\right\rangle= & \left\langle k_{f} \phi_{f}|V| \phi_{i} k_{i}\right\rangle \\
& +\sum_{n=1}^{N} \int d k \frac{\left\langle k_{f} \phi_{f}|V| \phi_{n} k\right\rangle\left\langle k \phi_{n}|T| \phi_{i} k_{i}\right\rangle}{E+i 0-\varepsilon_{n}-k^{2} / 2},
\end{aligned}
$$

where $E=\varepsilon_{n}+k_{n}^{2} / 2$ is the total energy, and $V$ is a combination of interaction potentials that depend on the wavefunction symmetry [21]. Note that for the Temkin-Poet model we can write the momenta $k$ as scalars also.

The key feature of the CCC method is that convergence in the results of interest should be observed with increasing number of states $N$ in the expansion of Eq. (3). This has the effect of increasing the number of coupled equations in Eq.(4), whose computational method of solution is specified in Ref. [21].

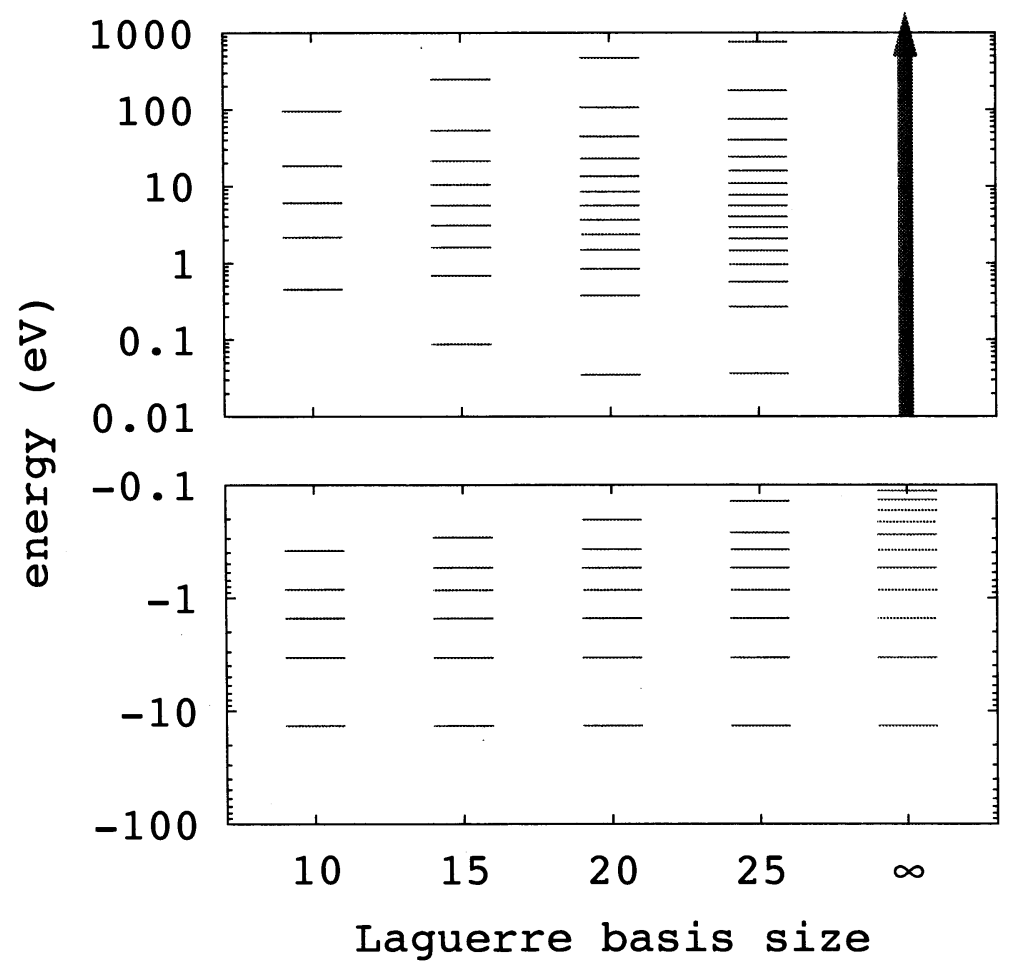

FIG. 3: Hydrogen $l=0$ energy levels in CCC-L calculations for $\lambda=1$ and specified basis size.

To check the convergence we shall consider here just the original CCC-L approach. In figure 3 we show what happens to the target-state energies as the Laguerre basis is increased, with both the discrete and the continuous spectra becoming more densely populated.

As discussed earlier, Temkin and Poet [4-9] gave a set of benchmark results for the e-H model problem. In testing the CCC method we need to ensure that convergence is obtained and that it is to the correct values given by Temkin and Poet. In figure 4 we present the results of three CCC calculations, for $N=5,10,30$. We see that for the smallest calculation there can be very large unphysical oscillations in the cross sections, particularly at the lower energies and for the higher transitions. As the size of the calculations increases the cross sections converge to a smooth result that is in good agreement with the benchmark results. The cross sections for the individual transitions are obtained 


\section{Symposium on Atomic \& Molecular Physics}

simply from the magnitudes of the corresponding $T$-matrix elements obtained from Eq. (4). The total ionization cross section is obtained as a sum of cross sections of all positive-energy states. Note that no comparison of the total ionization cross section was possible [23], but when CCC was applied to the full e-H problem [24] excellent agreement with experiment was obtained.

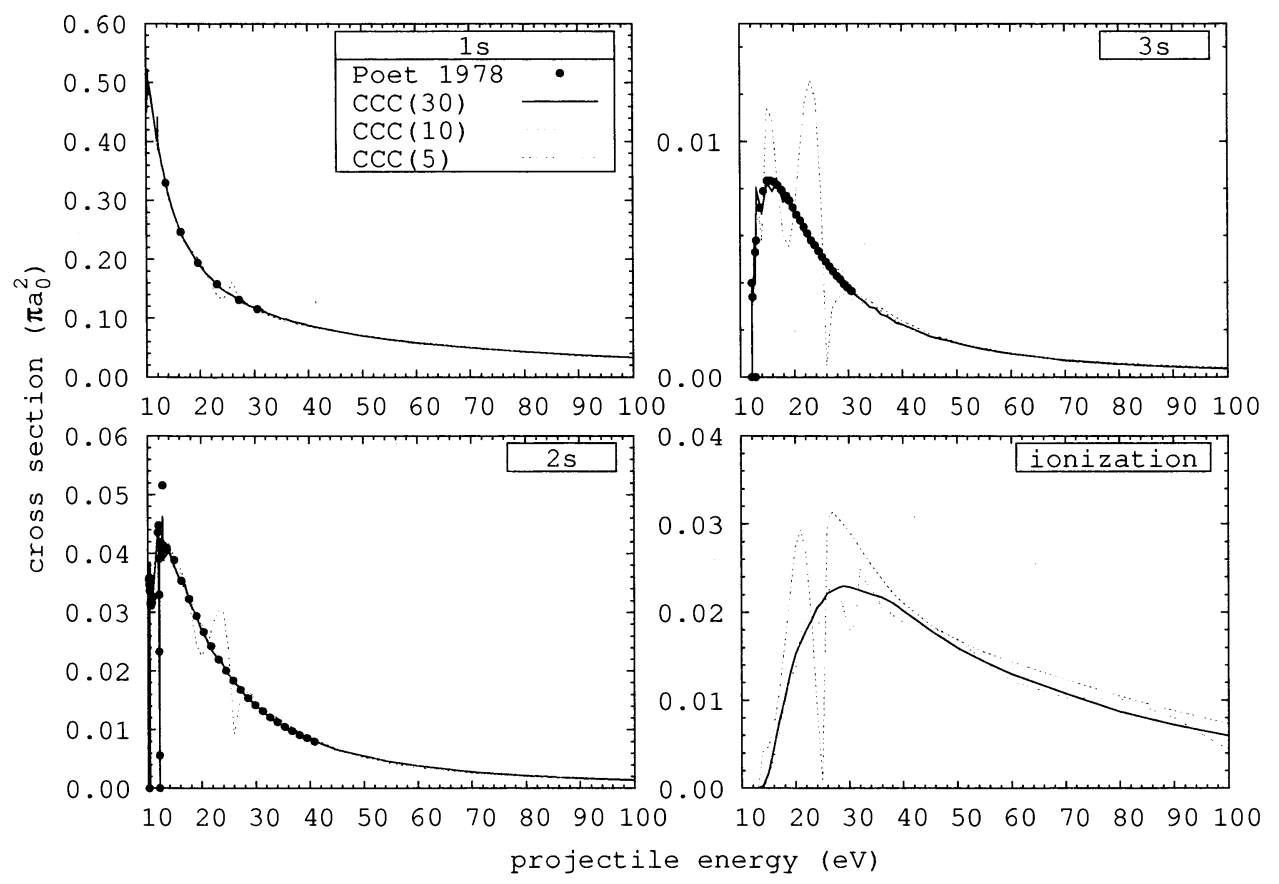

FIG. 4: Temkin-Poet model electron-hydrogen cross sections calculated with the CCC-L method using the specified basis sizes.

Having obtained an overall picture as a function of energy we now look a little closer at a specific incident electron energy of $3 \mathrm{Ry}(E=2 \mathrm{Ry})$. In figure 5 we present a convergence study for all negative-energy states arising in the CCC calculations of specified $N$. The first thing to note is that the largest cross sections converge first, and that the convergence appears to be from above. The least negative-energy state in each calculation reverse the diminishing cross section trend. These states are not true eigenstates and have the effect of summing the cross sections for all the negative-energy states not explicitly included in the calculations.

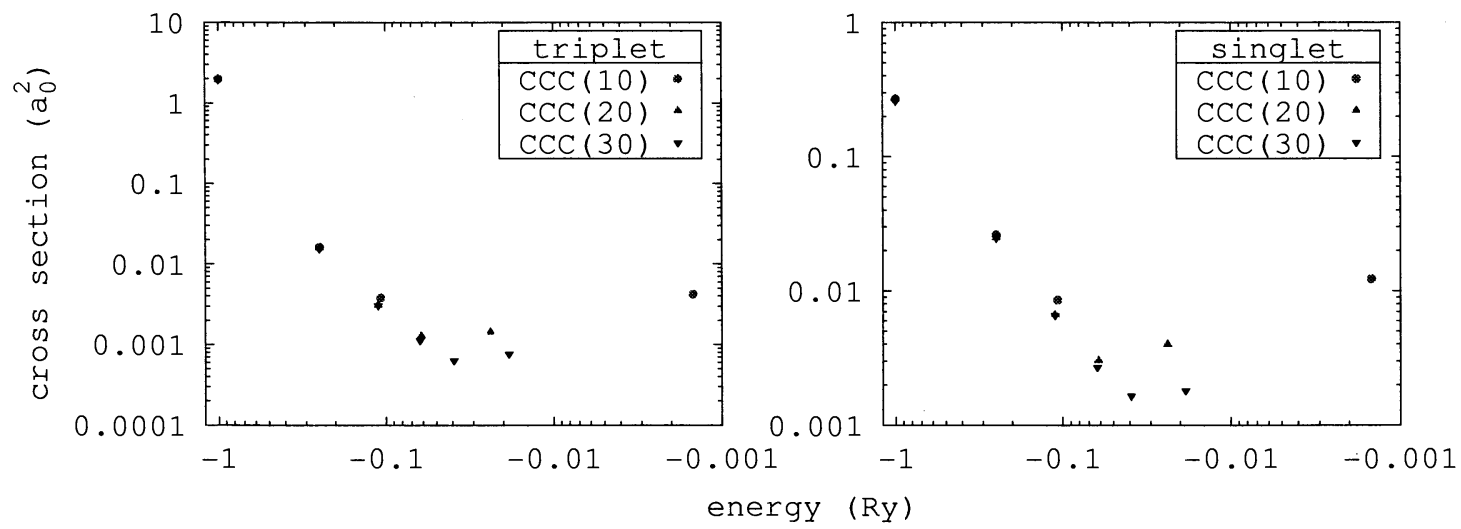

FIG. 5: Temkin-Poet model of 3 Ry e-H scattering. Negative-energy state cross sections calculated with the CCC method of specified basis size.

Successful reproduction of the Temkin-Poet model benchmark results was followed by application to the full e-H $2 \mathrm{p}$ excitation problem [21]. However, the CCC results were also unable to reproduce the experiment [12, 13], and were more in agreement with previous calculations [14-17]. Nevertheless, the sound foundations of the CCC method and 


\section{Symposium on Atomic \& Molecular Physics}

its numerous successes elsewhere [25, 26] motivated the experimentalists to revisit the problem [27, 28] yielding much better agreement with the theory.

Having found good agreement with the results of Temkin and Poet for discrete excitation, we realised that the model could be taken further and address some of the fundamental issues in the theory of electron-impact ionization. We have already demonstrated in figure 4 that at any energy above the ionization threshold the total ionization cross section converges with increasing basis size. Now we take a specific incident electron energy and ask the question does the underlying singly differential cross section converge also. In particular, we note from Eq. (4) that when summing over the positive-energy cross sections we sum over all open channels for which $0 \leq \varepsilon_{n} \leq E$. Since we have two identical electrons in the problem it appears that the identical ionization process with electron energies $e_{a}=\epsilon_{n}, e_{b}=E-\epsilon_{n}$ and $e_{a}=E-\epsilon_{n}, e_{b}=\epsilon_{n}$ is being counted twice. Yet the close coupling theory is unitary and does not allow for double-counting.
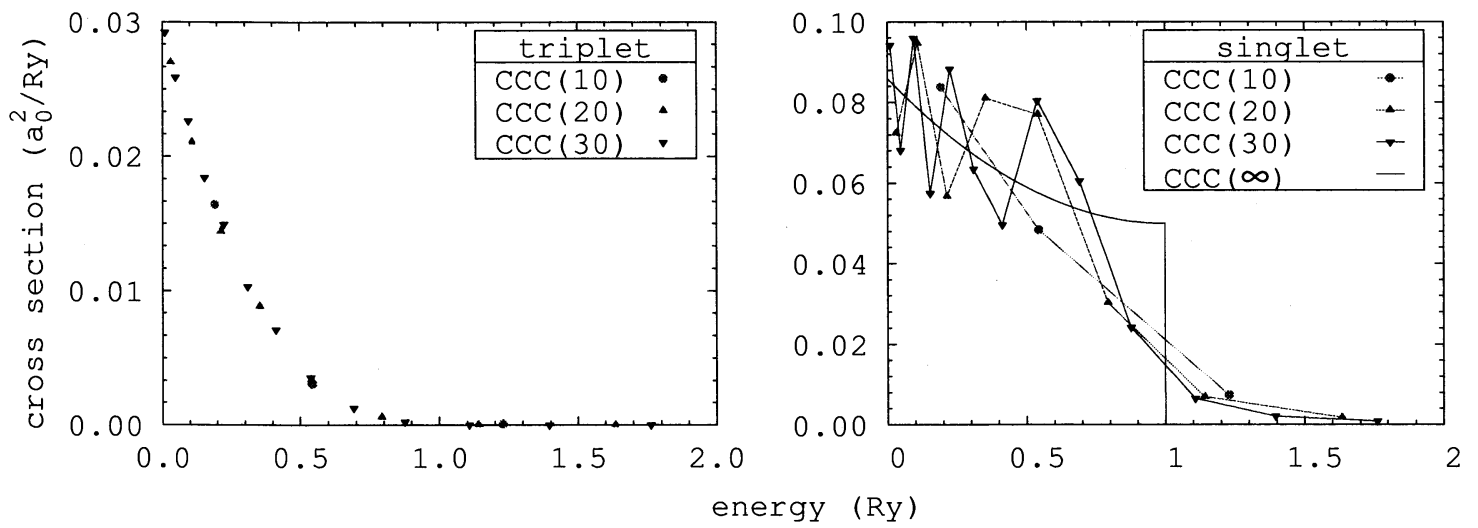

FIG. 6: Temkin-Poet model of 3 Ry e-H scattering. Positive-energy state cross sections calculated with the CCC method of specified basis size. The straight lines connecting the points are there to help guide the eye. The step-function denoted by $\mathrm{CCC}(\infty)$ is an integral preserving estimate.

In figure 6 we consider the same calculations as presented in figure 5 , but this time we look at the positive-energy state cross sections, which have been converted to singly differential cross sections (the integral, rather than the sum, yields the total ionization cross section of figure 4). Looking at the triplet case first, we see convergence to a smooth line which tends to zero at $1 \mathrm{Ry}$ (equal energy-sharing), and remains zero for larger energies. In the singlet case the situation is more complicated. Here we see substantial oscillation and apparently a lack of convergence, particularly at energies below 1 Ry. Observation of this behaviour suggested that the CCC-calculated SDCS is converging to a step function [29], and that solving Eq. (4) is like taking a Fourier expansion of a step function [30]. In this case at the step the underlying amplitudes should converge to half the true amplitude magnitude, and hence the cross section to one quarter of the true cross section. The $\operatorname{CCC}(\infty)$ estimate given in figure 6 was obtained this way. Subsequently, methods like the exterior complex scaling [31,32] showed very good agreement with the CCC estimates.

The notion that the CCC methods should yield amplitudes that are zero for $\epsilon_{n}>E / 2$ resolves the apparent problem with double-counting. In effect this reduces the endpoint of the SDCS integration from $E$ to $E / 2$, as would be expected in any theory that treats the two electrons identically. Application of these ideas to the full e-H and e-He ionization problems has resulted in excellent agreement with experiment $[33,34]$.

\section{CONCLUSIONS}

The Temkin-Poet model has played a crucial role in the development of general electron-atom scattering theories, and continues to do so. It retains the complexity associated with the infinite target discrete and continuous spectrum, as well as electron exchange. The unique nature of the underlying Schrödinger equation allows for an accurate solution leading to benchmark results against which general methods may be tested. It is helpful not only for discrete scattering, but also for ionization problems. The success of the convergent close-coupling method for electron-, photonand positron- scattering on atoms can all be traced back to the simple model problem first considered by Aaron Temkin 
back in 1962 .

[1] R. K. Nesbet, Phys. Rev. A 20, 58 (1979).

[2] P. G. Burke and W. D. Robb, Adv. Atom. Mol. Phys. 11, 143 (1975).

[3] D. H. Madison and W. N. Shelton, Phys. Rev. A 7, 499 (1973).

[4] A. Temkin, Phys. Rev. 126, 130 (1962).

[5] A. Temkin and E. Sullivan, Phys. Rev. 129, 1250 (1963).

[6] H. L. Kyle and A. Temkin, Phys. Rev. 129, A600 (1964).

[7] R. Poet, J. Phys. B 11, 3081 (1978).

[8] R. Poet, J. Phys. B 13, 2995 (1980).

[9] R. Poet, J. Phys. B 14, 91 (1981).

[10] I. A. Howard and N. H. March, Physics and chemistry of liquids 43, 559 (2005).

[11] M. J. Mitnik DM, J. Phys. B 38, 3325 (2005).

[12] E. Weigold, L. Frost, and K. J. Nygaard, Phys. Rev. A 21, 1950 (1980).

[13] J. F. Williams, J. Phys. B 14, 1197 (1981).

[14] J. Callaway, Phys. Rev. A 32, 775 (1985).

[15] W. L. van Wyngaarden and H. R. J. Walters, J. Phys. B 19, 929 (1986).

[16] D. H. Madison, I. Bray, and I. E. McCarthy, Phys. Rev. Lett. 64, 2265 (1990).

[17] T. T. Scholz, H. R. J. Walters, P. G. Burke, and M. P. Scott, J. Phys. B 24, 2097 (1991).

[18] M. B. Shah, D. S. Elliot, and H. B. Gilbody, J. Phys. B 20, 3501 (1987).

[19] R. G. Montague, M. F. A. Harrison, and A. C. H. Smith, J. Phys. B 17, 3295 (1984).

[20] M. B. Shah, D. S. Elliot, P. McCallion, and H. B. Gilbody, J. Phys. B 21, 2751 (1988).

[21] I. Bray and A. T. Stelbovics, Phys. Rev. A 46, 6995 (1992).

[22] I. Bray, K. Bartschat, and A. T. Stelbovics, Phys. Rev. A 67, 060704(R) (2003).

[23] I. Bray and A. T. Stelbovics, Phys. Rev. Lett. 69, 53 (1992).

[24] I. Bray and A. T. Stelbovics, Phys. Rev. Lett. 70, 746 (1993).

[25] I. Bray, Phys. Rev. A 49, 1066 (1994).

[26] D. V. Fursa and I. Bray, Phys. Rev. A 52, 1279 (1995).

[27] H. Yalim, D. Cvejanovic, and A. Crowe, Phys. Rev. Lett. 79, 2951 (1997).

[28] R. W. O'Neill, P. J. M. van der Burgt, D. Dziczek, P. Bowe, S. Chwirot, and J. A. Slevin, Phys. Rev. Lett. 80, 1630 (1998).

[29] I. Bray, Phys. Rev. Lett. 78, 4721 (1997).

[30] A. T. Stelbovics, Phys. Rev. Lett. 83, 1570 (1999).

[31] M. Baertschy, T. N. Rescigno, W. A. Isaacs, and C. W. McCurdy, Phys. Rev. A 60, R13 (1999).

[32] P. L. Bartlett and A. T. Stelbovics, Phys. Rev. A 69, 022703 (2004).

[33] I. Bray, Phys. Rev. Lett. 89, 273201 (2002).

[34] A. T. Stelbovics, I. Bray, D. V. Fursa, and K. Bartschat, Phys. Rev. A 71, 052716(13) (2005). 